\title{
EFECTO DE TÉCNICAS DE ESTABLECIMIENTO SOBRE EL DESARROLLO INICIAL DE PLANTACIONES DE ALGARROBO (Prosopis chilensis (Mol.) Stuntz) EN LA ZONA CENTRAL DE CHILE ${ }^{16}$
}

\author{
González, Marlene ${ }^{17}$; Hormazabal, Marco ${ }^{18}$ y Salinas, Aldo ${ }^{18}$
}

\section{RESUMEN}

Se presentan resultados de un conjunto de ensayos de técnicas de establecimiento de plantaciones con algarrobo (Prosopis chilensis), en una zona dentro de su distribución natural y donde actualmente es habitual su uso en programas de forestación o reforestación. Se incluye la evaluación de fertilización inicial, del uso de distinto tipo de protectores individuales contra daños por animales silvestre y doméstico, y del uso de dispositivos Cocoon® sobre el desarrollo inicial de las plantas.

Las primeras evaluaciones indican que hay efectos de significancia estadística, en todos los ensayos, lo que podría entregar indicadores para realizar mejoras en las técnicas utilizadas y de esa manera garantizar el desarrollo futuro de plantaciones con la especie, aumentando su probabilidad de éxito y uso tanto en programas de restauración como con fines productivos.

Palabras clave: Prosopis chilensis, algarrobo, Cocoon®

\section{SUMMARY}

Results of a set of plantation establishment techniques trials with Carob Tree (Prosopis chilensis) in the area of its natural distribution and where its use in afforestation and reforestation programs is usual are presented. The paper includes the evaluation of the effect of initial fertilization, different kind of individual protectors against wild and domestic animals, and the use of Cocoon $\AA$ devices on the initial development of these plants.

The first evaluations indicate that there are effects of statistical significance in all the trials, which could provide indicators to improve the techniques used and guarantee the future development of Carob Tree plantations, increasing its success probability to be used in restoration programs or productive purposes.

Keywords: Prosopis chilensis, Carob Tree, Cocoon ${ }^{\circledR}$

\footnotetext{
${ }^{16}$ Actividades financiadas por el Ministerio de Agricultura a través del área de investigación de INFOR Silvicultura y Manejo de Ecosistemas Forestales Nativos y Exóticos.

17 Ingeniero Forestal. Instituto Forestal, Sede Metropolitana. marlene.gonzalez@infor.cl

${ }^{18}$ Técnicos. Instituto Forestal, Sede Metropolitana. mhormazabal@infor.cl; asalinas@infor.cl
} 


\section{INTRODUCCIÓN}

Prosopis chilensis (Mol.) Stuntz es la especie de este género botánico de más amplia distribución en Chile, se la puede encontrar desde la región de Tarapacá hasta la de O'Higgins, pero más del $50 \%$ de la superficie nacional de la especie se concentra en la región Metropolitana (INFOR, 1986), alcanzando al año 2013 a 11.828 ha (González, 2013).

La especie crece naturalmente en valles o cuencas con napas freáticas relativamente superficiales, en fondos de quebradas, donde la acumulación hídrica se hace más efectiva, con ejemplares dispersos o formando pequeños bosquetes. Se la puede encontrar en el Valle Central, en la parte inferior del piedemonte de la Cordillera de la Costa y en quebradas de la Cordillera de la Costa y de los cordones transversales (Altamirano, 2012) y siempre alejado de la influencia marina (Martinez, 1998).

Como a todas las especies de Prosopis, al algarrobo se le reconocen características particulares, como desarrollarse en regiones con déficit hídrico permanente, en suelos deficitarios en nitrógeno, en condiciones de alta intensidad de radiación solar y con grandes variaciones de temperatura entre el día y la noche (Balboa et al., 1988).

Estos mismos atributos, han motivado que FAO haya reconocido a los algarrobos como un importante recurso para las poblaciones humanas que dependen de la agricultura de subsistencia, tanto así que su cultivo ha sido recomendado para combatir la desertificación y recuperar ecosistemas degradados (Bernuy, 2003).

Respecto de su presencia histórica, es posible encontrar importantes menciones de la especie respecto de su participación en el paisaje y las actividades cotidianas de la población en esta área geográfica a través del tiempo y de su uso particular asociado a la producción de ganado, la agricultura y como combustible.

Esto ha significado que la densidad de la especie en formaciones naturales muestre una disminución, explicada principalmente por la sobrexplotación de su madera para obtención de leña, construcción de viviendas, fundición de metales y elaboración de carbón.

Este recurso también ha sido afectado por la habilitación de terrenos para uso agrícola y además la especie está sometida a presiones por el avance de proyectos inmobiliarios, poniéndola en un grado de vulnerabilidad importante (Pinto y Riveros, 1989, citados por Caro et al., 1991).

Valdivia y Romero (2013) indican que la depredación de semillas y plantas por animales silvestres (conejos y liebres) y domésticos (ganado bovino y caprino) también ha contribuido a la degradación del algarrobo y a la reestructuración del bosque espinoso, ya que el paso por el tracto digestivo de los animales actuaría como una limitante en su capacidad germinativa, limitando su regeneración en forma natural.

La suma de estas condiciones se ha traducido en la incorporación de Prosopis chilensis en forma habitual en planes de forestación y reforestación de la zona central del país, la mayoría asociados a compromisos de compensación por obras civiles, pero el éxito de dichas plantaciones no ha sido el esperado, ya que es común observar que con la finalidad de cumplir con las exigencias legales, las plantas son sometidas a intervenciones intensivas, especialmente riego permanente, pero solo durante el periodo inicial de fiscalización, para posteriormente ser abandonadas sin lograrse así el efectivo establecimiento de la plantación. 
El objetivo de este estudio es probar técnicas que incidan positivamente en el éxito de las plantaciones y que permitan promover intervenciones de establecimiento adecuadas, de manera de frenar el proceso de degradación actual y lograr un grado de productividad que permita sostener los sistemas agroforestales y silvopastorales que se han mantenido a lo largo de la historia en estas regiones y que podrían volver a convertirse en parte de las actividades de la población de estos territorios.

\section{MATERIAL Y MÉTODO}

El conjunto de ensayos fue establecido en el predio Quilapilún, comuna de Colina, provincia de Chacabuco, región Metropolitana (Coordenadas UTM (19H) 345412 - 6337171, Altitud $804 \mathrm{msnm}$ ), zona que es representativa del área de distribución natural de Prosopis chilensis. En el año 2013 se instaló el ensayo de fertilización inicial, en 2014 el de protectores individuales y finalmente en 2016 el de los dispositivos Cocoon \$. Todos ellos dentro de un cerco perimetral común para evitar el daño de lagomorfos y animales domésticos. Para la construcción de este cerco se utilizaron postes impregnados de 3" de diámetro y 2,44 m de largo, malla hexagonal de 1" y $1,2 \mathrm{~m}$ de altura, enterrada $5 \mathrm{~cm}$. Adicionalmente, se instalaron tres hebras de alambre de púas en la parte alta para impedir ingreso de animales mayores (Figura $\mathrm{N}^{\circ} 1$ ).

\section{Fertilización Inicial}

Durante junio del año 2013 se instaló el ensayo estableciendo 100 plantas de algarrobo de dos temporadas de vivero (2:M) producidas en el vivero de la Universidad de Chile en Antumapu en macetas de polietileno de $20 \times 30 \mathrm{~cm}$, distribuidas en dos tratamientos (Cuadro $\mathrm{N}^{\circ} 1$ ), en casillas de $40 \times 40 \times 40 \mathrm{~cm}$, con espaciamiento de $3 \times 3 \mathrm{~m}$, en las que se construyeron tasas de riego con capacidad aproximada de $20 \mathrm{~L}$ de agua por casilla. El objetivo es evaluar el efecto de la fertilización sobre el desarrollo inicial de las plantas.
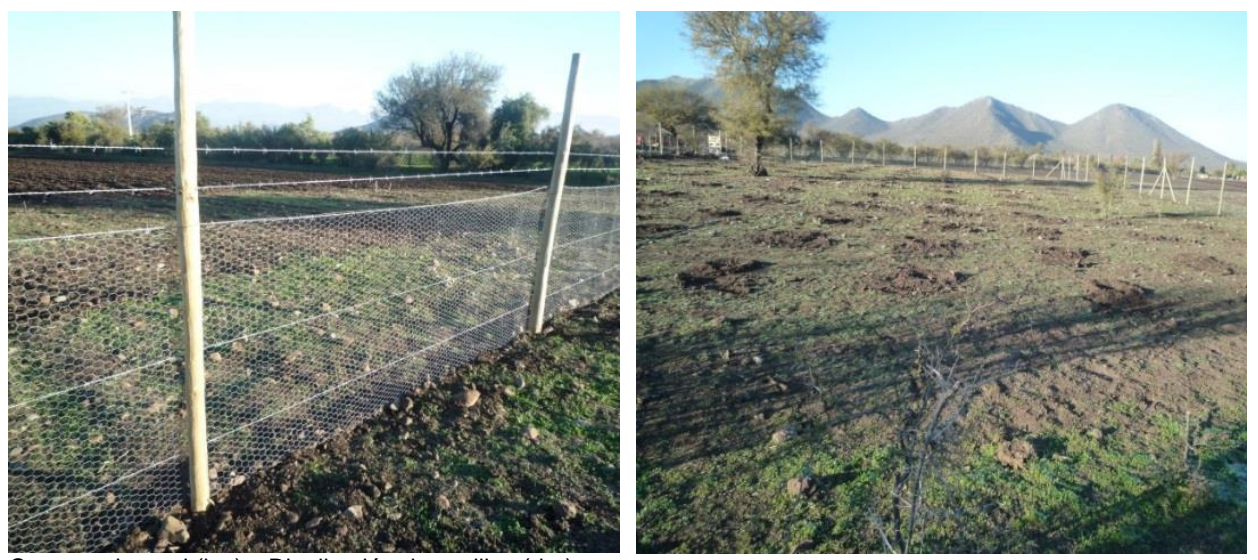

Cerco perimetral (izq) y Distribución de casillas (der)

Figura $N^{\circ} 1$

VISTA GENERAL DEL ENSAYO 
Cuadro $\mathrm{N}^{\circ} 1$

TRATAMIENTOS DE FERTILIZACIÓN INICIAL

\begin{tabular}{|c|l|c|}
\hline Tratamiento & \multicolumn{1}{|c|}{ Fertilizante Utilizado } & $\begin{array}{c}\text { Plantas } \\
\text { Instaladas } \\
\left(\mathbf{N}^{\circ} \mathbf{)}\right.\end{array}$ \\
\hline $\mathrm{T} 1$ & Testigo sin aplicación & 50 \\
\hline $\mathrm{T} 2$ & $70 \mathrm{~g}$ NPK y $3 \mathrm{~g}$ Gel Hidratante & 50 \\
\hline
\end{tabular}

El gel fue distribuido uniformemente al plantar en torno y bajo el pan de tierra que cubre la raíz de la planta, y el fertilizante fue aplicado en pequeñas zanjas practicadas a $10 \mathrm{~cm}$ alrededor de la planta inmediatamente después de la plantación.

Dada la condición de sequía declarada en la provincia de Chacabuco durante el año 2014, se mantuvo la aplicación de riego a la totalidad de las plantas, con una dosis de $20 \mathrm{~L} /$ planta, según el calendario indicado en Cuadro $\mathrm{N}^{\circ} 2$.

\section{Cuadro $\mathrm{N}^{\circ} 2$}

FECHAS DE APLICACIÓN DE RIEGO

\begin{tabular}{|c|l|}
\hline Riego & Fecha \\
\hline 1 & $02 /$ Ene \\
\hline 2 & $28 /$ Ene \\
\hline 3 & $28 /$ Feb \\
\hline 4 & $20 / \mathrm{Mar}$ \\
\hline 5 & $29 / \mathrm{Abr}$ \\
\hline 6 & $26 / \mathrm{May}$ \\
\hline 7 & $27 / \mathrm{Nov}$ \\
\hline 8 & $22 / \mathrm{Dic}$ \\
\hline
\end{tabular}

Para la evaluación del efecto de los tratamientos sobre el desarrollo inicial de las plantas se midió las variables diámetro a la altura del cuello DAC $(\mathrm{mm})$, altura total del vástago principal (cm) y mortalidad, durante el establecimiento y las tres temporadas posteriores (Cuadro $\mathrm{N}^{\circ} 3$ ).

\section{Cuadro $\mathrm{N}^{\circ} 3$}

VARIABLES EVALUADAS POR TRATAMIENTO SEGÚN TEMPORADA

\begin{tabular}{|c|c|c|c|c|c|c|c|c|c|}
\hline \multirow{2}{*}{ Tratamiento } & \multirow{2}{*}{$\begin{array}{c}\text { Plantas } \\
\text { Establecidas } \\
\left(\mathbf{N}^{\circ}\right)\end{array}$} & \multicolumn{4}{|c|}{$\begin{array}{l}\text { Altura Vástago Principal } \\
\text { (cm) }\end{array}$} & \multicolumn{4}{|c|}{$\begin{array}{l}\text { DAC } \\
(\mathrm{mm})\end{array}$} \\
\hline & & $\begin{array}{l}\text { Jun } \\
2013\end{array}$ & $\begin{array}{l}\text { Sep } \\
2014\end{array}$ & $\begin{array}{l}\text { Jun } \\
2015\end{array}$ & $\begin{array}{l}\text { Nov } \\
2016\end{array}$ & $\begin{array}{l}\text { Junio } \\
2013\end{array}$ & $\begin{array}{l}\text { Sep } \\
2014\end{array}$ & $\begin{array}{l}\text { Jun } \\
2015\end{array}$ & $\begin{array}{l}\text { Nov } \\
2016\end{array}$ \\
\hline $\mathrm{T} 1$ & 50 & $x$ & $x$ & $x$ & $x$ & $x$ & $x$ & $x$ & $x$ \\
\hline T2 & 50 & $x$ & $x$ & $x$ & $x$ & $x$ & $x$ & $x$ & $x$ \\
\hline
\end{tabular}




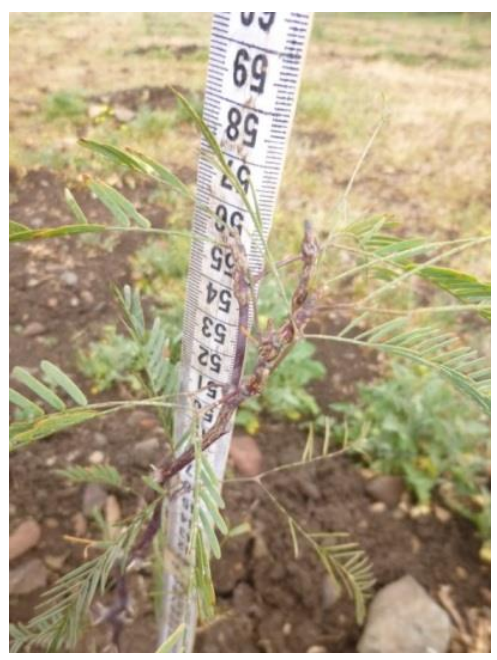

Figura $\mathrm{N}^{\circ} 2$

MEDICIÓN DE LA ALTURA TOTAL DEL VÁSTAGO PRINCIPAL

\section{Protectores Individuales}

En Chile, las plantaciones pueden sufrir grandes daños debido al ataque por liebres, conejos y roedores, lo que puede traducirse en un completo fracaso de los proyectos de forestación y reforestación, especialmente en zonas secas. Estos animales comen las plantas o parte de ellas, las cortan o las quiebran. Para evitar o minimizar este daño, es habitual proteger las plantas excluyendo las zonas de plantación o utilizando barreras químicas o físicas para cada planta.

En agosto del 2014, a un costado del ensayo anteriormente descrito, se instalaron dos nuevas parcelas, destinadas a evaluar dos tipos de protectores individuales y su efecto sobre el desarrollo de plantas de algarrobo, al prevenir el daño causado por animales silvestres (Figura $\mathrm{N}^{\circ}$ 3 ). Dichos tratamientos se detallan en Cuadro $\mathrm{N}^{\circ} 4$. Se empleó plantas producidas en macetas de polietileno $(1: \mathrm{M})$ y el espaciamiento fue de $3 \times 3 \mathrm{~m}$. Este ensayo también recibió riegos periódicos inicialmente.

\section{Cuadro $\mathrm{N}^{\circ} 4$}

TRATAMIENTOS DE PROTECCIÓN INDIVIDUAL

\begin{tabular}{|c|c|c|}
\hline Tratamiento & Tipo de Protector & $\begin{array}{c}\text { Plantas Establecidas } \\
\left(\mathbf{N}^{\circ}\right)\end{array}$ \\
\hline T1 & Malla Raschel con coligües & 28 \\
\hline T2 & Tetrapack & 32 \\
\hline
\end{tabular}




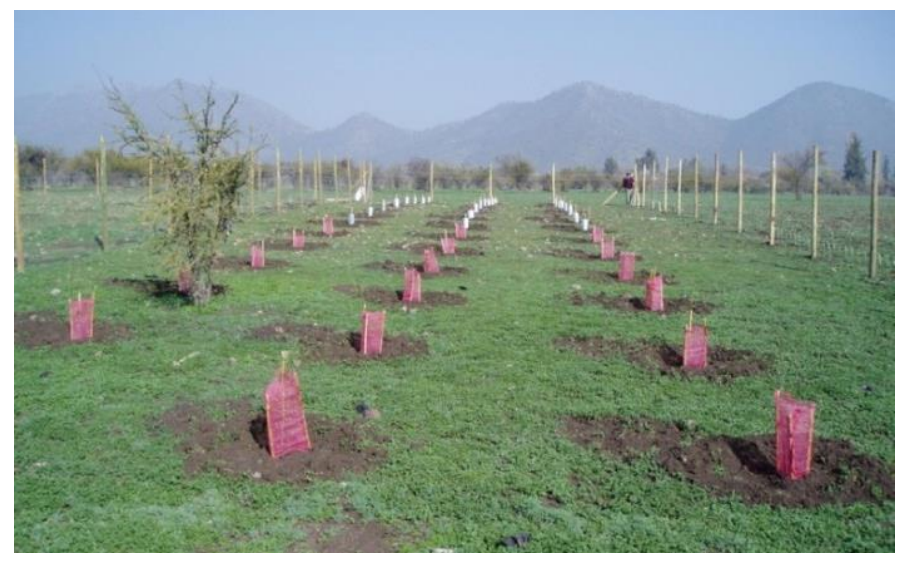

Figura $\mathrm{N}^{\circ} 3$

VISTA GENERAL DEL ENSAYO

Hasta ahora se han realizado tres evaluaciones, en todas ellas se registró para cada planta DAC diámetro a la altura del cuello $(\mathrm{mm})$, altura del vástago principal $(\mathrm{m})$ y el número de plantas muertas por tratamiento (Cuadro $\mathrm{N}^{\circ} 5$ ).

Cuadro $\mathrm{N}^{\circ} 5$

VARIABLES EVALUADAS POR TRATAMIENTO SEGÚN TEMPORADA

\begin{tabular}{|c|c|c|c|c|c|c|c|}
\hline \multirow{2}{*}{ Tratamiento } & \multirow{2}{*}{$\begin{array}{c}\text { Plantas } \\
\text { Establecidas } \\
\left(\mathbf{N}^{\circ}\right)\end{array}$} & \multicolumn{3}{|c|}{$\begin{array}{c}\text { Altura } \\
\text { (cm) }\end{array}$} & \multicolumn{3}{|c|}{$\begin{array}{l}\text { DAC } \\
(\mathrm{mm})\end{array}$} \\
\hline & & $\begin{array}{c}\text { Dic } \\
2014\end{array}$ & $\begin{array}{l}\text { Jun } \\
2015\end{array}$ & $\begin{array}{c}\text { Dic } \\
2016\end{array}$ & $\begin{array}{c}\text { Dic } \\
2014\end{array}$ & $\begin{array}{c}\text { Jun } \\
2015\end{array}$ & $\begin{array}{c}\text { Dic } \\
2016\end{array}$ \\
\hline T1 & 28 & $x$ & $x$ & $x$ & $x$ & $x$ & $x$ \\
\hline T2 & 32 & $x$ & $x$ & $x$ & $x$ & $x$ & $x$ \\
\hline
\end{tabular}

\section{Dispositivos Cocoon $®$}

En el año 2016, se estableció el último de los ensayos, consistente en una plantación utilizando el Cocoon $\AA$, dispositivo no probado anteriormente, diseñado por la empresa holandesa Landlife Company para plantaciones, especialmente en zonas áridas, capaz de mantener aproximadamente $25 \mathrm{~L}$ de agua acumulada en su interior por un periodo de tiempo prolongado.

El dispositivo funciona a través de capilaridad, utilizando dos cordones de algodón que entregan el agua en forma racionada, incluye un tubo protector contra conejos y no es reutilizable dado que está construido con material biodegradable, lo que es una ventaja respecto a otros dispositivos de este tipo, ya que no requiere su retiro posterior.

Las plantas utilizadas en esta parcela fueron producidas en speedling trays $\left(280 \mathrm{~cm}^{3}\right)$ por 
el Centro Tecnológico de la Planta Forestal (CTPF) de INFOR, a partir de semillas colectadas en febrero del año 2015, en Polpaico, comuna de Til Til, región Metropolitana.

Esta decisión está enmarcada en los lineamientos que entrega FAO en su promoción a la restauración y rehabilitación de ecosistemas, respecto de utilizar material genético apropiado, velando por la cercanía del lugar de colecta de semillas al lugar de plantación, como una forma de aumentar las probabilidades de éxito de la misma, por la adaptación de esos árboles a las condiciones del sitio de plantación.

Estas parcela fueron establecidas en octubre del 2016, con 52 plantas, a un espaciamiento de $3 \times 3 \mathrm{~m}$, siendo necesario realizar una holladura circular de $35 \mathrm{~cm}$ de profundidad y diámetro medio de $70 \mathrm{~cm}$ para el emplazamiento del dispositivo (Figuras $\mathrm{N}^{\circ} 4$ y $\mathrm{N}^{\circ} 5$ ).

Luego se niveló el fondo de la casilla y se introdujo el Cocoon®, quedando este a nivel de suelo. Posteriormente, se estabilizó con suelo mullido por todo el contorno y se agregó el agua hasta dos centímetros del borde superior.

En la parte central se estableció la planta dejando los cordones de algodón en contacto con la maceta de tierra, se instalaron las tapas y protectores contra conejos y finalmente se cubrió con una fina capa de suelo de manera de dar una mayor estabilidad al dispositivo.

Las evaluaciones en este caso se realizaron con periodicidad semestral, contándose hasta ahora con la medición inicial y dos posteriores, realizadas en abril y octubre del año 2017 (Cuadro $\mathrm{N}^{\circ} 6$ ).
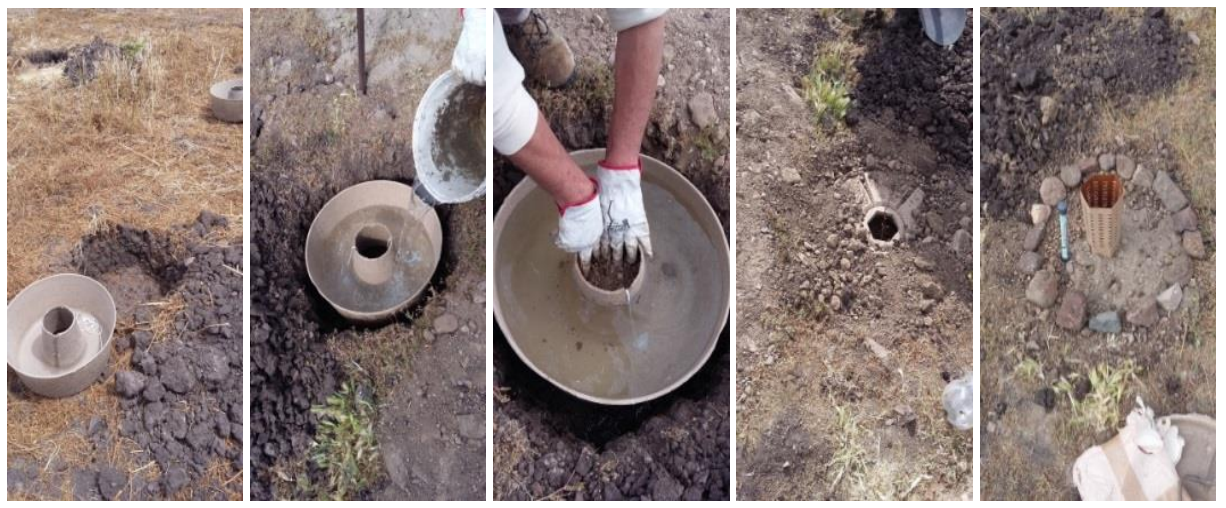

Figura $\mathrm{N}^{\circ} 4$

ETAPAS PARA INSTALACIÓN DE LOS DISPOSITIVOS COCOON® 


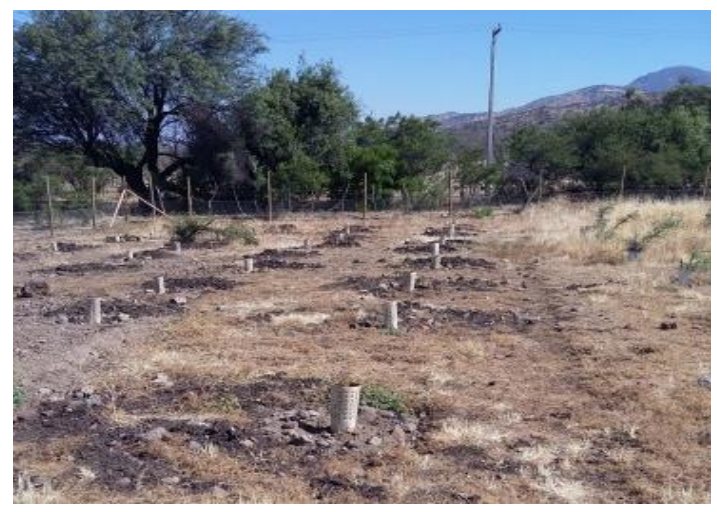

Figura $N^{\circ} 5$

VISTA GENERAL DEL ENSAYO CON DISPOSITIVOS COCOON®

\section{Cuadro $\mathrm{N}^{\circ} 6$}

VARIABLES EVALUADAS EN PLANTACIÓN CON DISPOSITIVO COCOON®

\begin{tabular}{|c|c|c|c|c|c|c|c|c|c|}
\hline \multirow{2}{*}{ Tratamiento } & \multirow{2}{*}{$\begin{array}{c}\text { Plantas } \\
\text { Establecidas } \\
\left(\mathbf{N}^{\circ}\right)\end{array}$} & \multicolumn{3}{|c|}{$\begin{array}{l}\text { Altura } \\
\text { (cm) }\end{array}$} & \multicolumn{3}{|c|}{$\begin{array}{l}\text { DAC } \\
(\mathrm{mm})\end{array}$} & \multicolumn{2}{|c|}{$\begin{array}{c}\text { Mortalidad } \\
(\%)\end{array}$} \\
\hline & & $\begin{array}{l}\text { Nov } \\
2016\end{array}$ & $\begin{array}{c}\text { Abr } \\
2017\end{array}$ & $\begin{array}{c}\text { Oct } \\
2017\end{array}$ & $\begin{array}{l}\text { Nov } \\
2016\end{array}$ & $\begin{array}{c}\text { Abr } \\
2017\end{array}$ & $\begin{array}{c}\text { Oct } \\
2017\end{array}$ & $\begin{array}{c}\text { Abr } \\
2017\end{array}$ & $\begin{array}{r}\text { Oct } \\
2017\end{array}$ \\
\hline $\mathrm{T} 1$ & 52 & $x$ & $x$ & $x$ & $x$ & $x$ & $x$ & $x$ & $x$ \\
\hline
\end{tabular}

\section{RESULTADOS}

\section{Fertilización Inicial}

Como era esperable, el tratamiento con fertilizante produjo un efecto positivo sobre el desarrollo inicial de las plantas, las medias de altura y DAC son mayores que en el testigo sin fertilización y la mortalidad de plantas es mínima ( $1,1 \%$ sumados ambos tratamientos). Los valores medios por tratamiento se incluyen en Cuadro $\mathrm{N}^{\circ} 7$.

\section{Cuadro $\mathrm{N}^{\circ} 7$}

VALORES MEDIOS DE VARIABLES EVALUADAS POR TEMPORADA SEGÚN TRATAMIENTO

\begin{tabular}{|c|c|c|c|c|c|c|c|c|c|c|}
\hline \multirow{2}{*}{ Tratamiento } & \multirow{2}{*}{$\begin{array}{c}\text { Plantas } \\
\text { Establecidas } \\
\left(\mathbf{N}^{\circ}\right)\end{array}$} & \multirow{2}{*}{$\begin{array}{c}\text { Plantas } \\
\text { Evaluadas }^{*} \\
\left(\mathbf{N}^{\circ}\right)\end{array}$} & \multicolumn{4}{|c|}{$\begin{array}{c}\text { Altura } \\
\text { (cm) }\end{array}$} & \multicolumn{4}{|c|}{$\begin{array}{c}\text { DAC } \\
(\mathrm{mm})\end{array}$} \\
\hline & & & $\begin{array}{c}\text { Jun } \\
2013\end{array}$ & $\begin{array}{l}\text { Sep } \\
2014\end{array}$ & $\begin{array}{l}\text { Jun } \\
2015\end{array}$ & $\begin{array}{l}\text { Nov } \\
2016\end{array}$ & $\begin{array}{c}\text { Jun } \\
2013\end{array}$ & $\begin{array}{l}\text { Sep } \\
2014\end{array}$ & $\begin{array}{l}\text { Jun } \\
2015\end{array}$ & $\begin{array}{l}\text { Nov } \\
2016\end{array}$ \\
\hline T1 & 50 & 28 & 51,8 & 58,0 & 49,9 & 58,2 & 4,6 & 9,6 & 11,1 & 17,0 \\
\hline $\mathrm{T} 2$ & 50 & 40 & 51,1 & 66,9 & 67,0 & 81,9 & 5,2 & 13,1 & 16,2 & 23,1 \\
\hline
\end{tabular}

*No se incluyeron en la evaluación plantas que se consideró estaban afectadas por otra vegetación surgida en el lugar o por la sombra de árboles más grandes. 
Cuadro $\mathbf{N}^{\circ} 8$

INCREMENTOS MEDIOS DE VARIABLES EVALUADAS POR TRATAMIENTO SEGÚN TEMPORADA

\begin{tabular}{|c|c|c|c|c|c|c|c|c|}
\hline \multirow{2}{*}{ Tratamiento } & \multirow{2}{*}{$\begin{array}{c}\text { Plantas } \\
\text { Establecidas } \\
\left(\mathrm{N}^{\circ}\right)\end{array}$} & \multirow{2}{*}{$\begin{array}{c}\text { Plantas } \\
\text { Evaluadas } \\
\left(\mathrm{N}^{\circ}\right)\end{array}$} & \multicolumn{3}{|c|}{$\begin{array}{l}\text { Altura* }^{*} \\
\text { (cm) }\end{array}$} & \multicolumn{3}{|c|}{$\begin{array}{l}\text { DAC* } \\
\text { (mm) }\end{array}$} \\
\hline & & & $\begin{array}{c}\text { Incr } \\
2013-14\end{array}$ & $\begin{array}{c}\text { Incr } \\
2014-15\end{array}$ & $\begin{array}{c}\operatorname{Incr} \\
2015-16\end{array}$ & $\begin{array}{c}\text { Incr } \\
2013-14\end{array}$ & $\begin{array}{c}\operatorname{Incr} \\
2014-15\end{array}$ & $\begin{array}{c}\text { Incr } \\
2015-16\end{array}$ \\
\hline $\mathrm{T} 1$ & 50 & 28 & 6,18 a & $-8,14$ a & 8,36 a & 5,02 a & 1,52 a & 5,89 a \\
\hline $\mathrm{T} 2$ & 50 & 40 & $15,82 \mathrm{~b}$ & 0,10 a & $14,90 \mathrm{a}$ & 7,86 b & $3,10 \mathrm{~b}$ & $6,88 a$ \\
\hline
\end{tabular}

* Test LSD Fischer, $\alpha=0,05$. Letras distintas indican diferencias significativas

En el Cuadro $\mathrm{N}^{\circ} 8$ y en la Figura $\mathrm{N}^{\circ} 6$ se presentan los incrementos en altura obtenidos durante tres temporadas evaluadas. Es posible observar que en general las plantas que recibieron fertilización (T2) muestran un incremento mayor durante todas las temporadas si se comparan con las plantas no tratadas (T1), no obstante que todas las plantas sufrieron un daño apical, producto del ataque de animales. Sin embargo, estas diferencias son significativas solo durante la primera temporada.
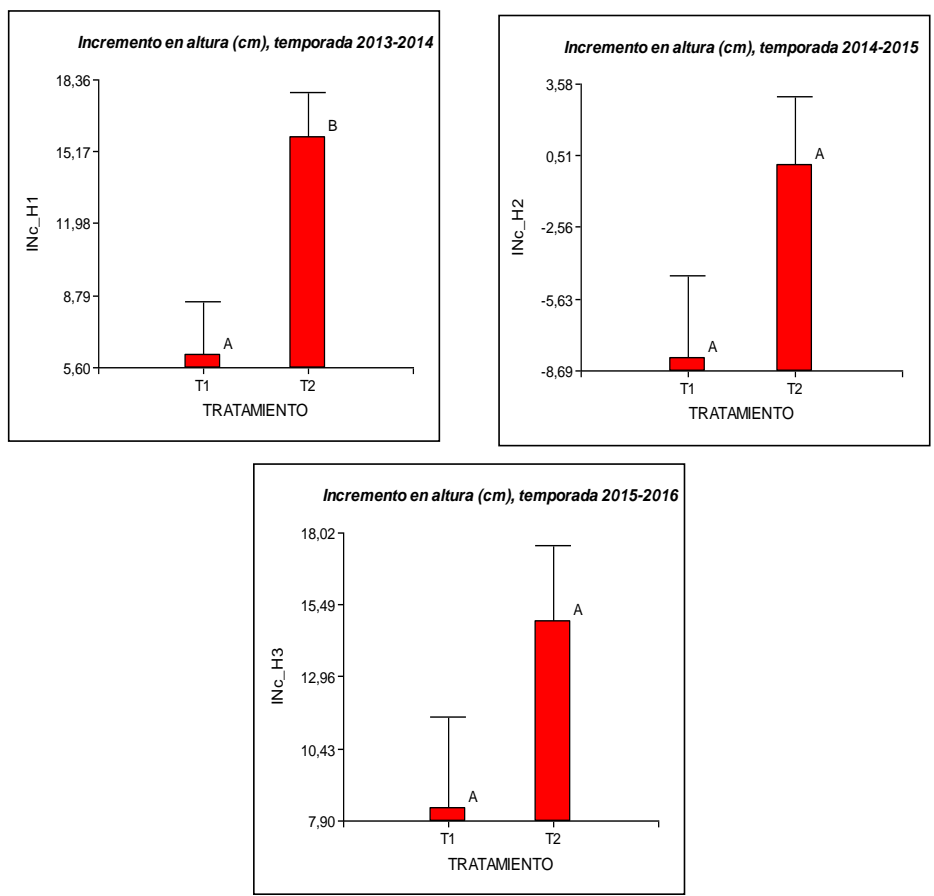

Figura $\mathrm{N}^{\circ} 6$

INCREMENTO EN ALTURA SEGÚN TRATAMIENTO 
Respecto del incremento en DAC, la tendencia se mantiene, son mayores los incrementos para las plantas fertilizadas (T2) y en este caso las diferencias son significativas hasta la segunda temporada (Figura $\mathrm{N}^{\circ} 7$ ).
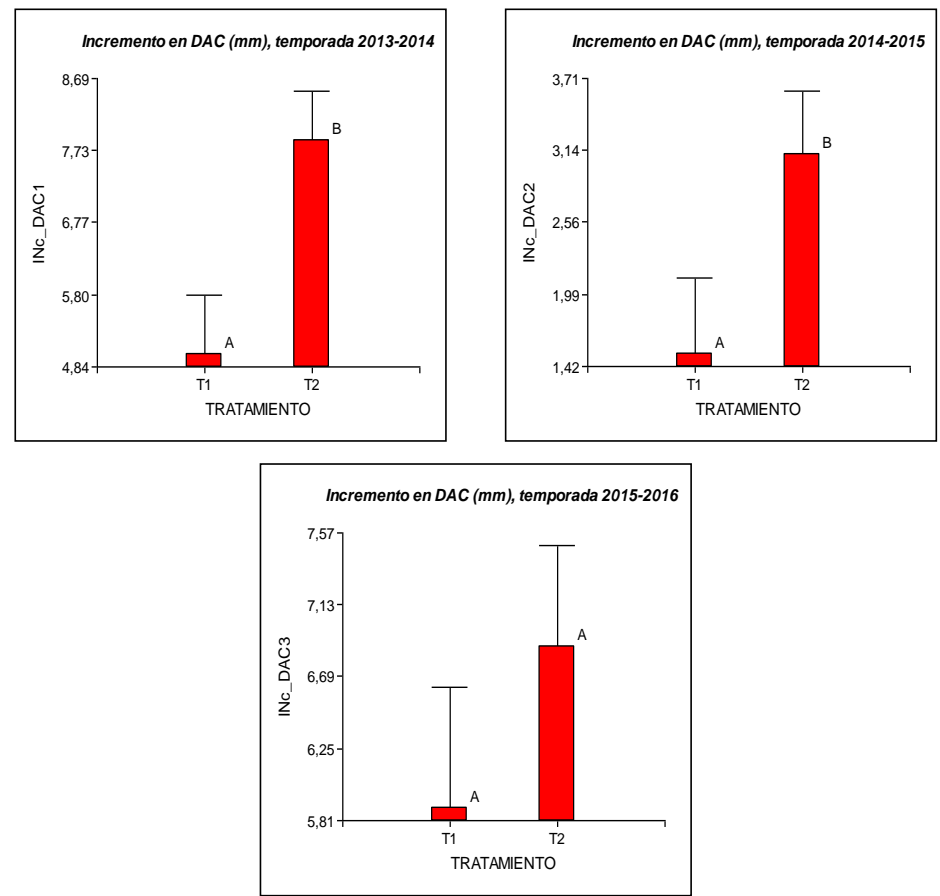

Figura $\mathbf{N}^{\circ} 7$

INCREMENTO EN DAC, SEGÚN TRATAMIENTO

\section{Protectores Individuales}

Realizadas dos evaluaciones y considerando solo las plantas supervivientes después de las tres temporadas y sin influencia de árboles mayores, es posible indicar que los protectores tuvieron un efecto sobre la disminución del daño, crecimiento y supervivencia de las plantas (Cuadro $\mathrm{N}^{\circ}$ 9).

Todas las plantas fueron ramoneadas por conejos sobre la altura de los protectores lo que indica la necesidad de reconsiderar la altura de estos para próximas plantaciones, ya que con seguridad las plantas serán nuevamente dañadas una vez que se recuperen y generen nuevos brotes, material aún más atractivo para los animales.

El daño no afecta los resultados dado que fue homogéneo para ambos tratamientos. 


\section{Cuadro $N^{\circ} 9$}

VALORES MEDIOS DE VARIABLES EVALUADAS POR TEMPORADA SEGÚN TRATAMIENTO

\begin{tabular}{|c|c|c|c|c|c|c|c|c|}
\hline \multirow{2}{*}{ Tratamiento } & \multirow{2}{*}{$\begin{array}{c}\text { Plantas } \\
\text { Establecidas } \\
\left(\mathrm{N}^{\circ}\right)\end{array}$} & \multirow{2}{*}{$\begin{array}{c}\text { Plantas } \\
\text { Evaluadas } \\
\left(\mathbf{N}^{\circ}\right)\end{array}$} & \multicolumn{3}{|c|}{$\begin{array}{c}\text { Altura } \\
\text { (cm) }\end{array}$} & \multicolumn{3}{|c|}{$\begin{array}{l}\text { DAC } \\
(\mathrm{mm})\end{array}$} \\
\hline & & & $\begin{array}{c}\text { Dic } \\
2014 \\
\end{array}$ & $\begin{array}{c}\text { Jun } \\
2015 \\
\end{array}$ & $\begin{array}{c}\text { Dic } \\
2016 \\
\end{array}$ & $\begin{array}{c}\text { Dic } \\
2014 \\
\end{array}$ & $\begin{array}{c}\text { Jun } \\
2015 \\
\end{array}$ & $\begin{array}{c}\text { Dic } \\
2016 \\
\end{array}$ \\
\hline $\mathrm{T} 1$ & 28 & 26 & 40,6 & 37,1 & 70,3 & 5,6 & 12,1 & 5,9 \\
\hline T2 & 32 & 27 & 40,7 & 42,8 & 99,2 & 6,4 & 17,3 & 7,0 \\
\hline
\end{tabular}

Cuadro $N^{\circ} 10$

INCREMENTOS MEDIOS DE VARIABLES EVALUADAS POR TEMPORADA SEGÚN TRATAMIENTO

\begin{tabular}{|c|c|c|c|c|c|c|}
\hline \multirow{2}{*}{ Tratamiento } & \multirow{2}{*}{$\begin{array}{c}\text { Plantas } \\
\text { Establecidas } \\
\left(\mathbf{N}^{\circ}\right)\end{array}$} & \multirow{2}{*}{$\begin{array}{c}\text { Plantas } \\
\text { Evaluadas } \\
\left(\mathbf{N}^{\circ}\right)\end{array}$} & \multicolumn{2}{|c|}{$\begin{array}{c}\text { Altura } \\
\text { (cm) }\end{array}$} & \multicolumn{2}{|c|}{$\begin{array}{l}\text { DAC } \\
(\mathrm{mm})\end{array}$} \\
\hline & & & $\begin{array}{c}\text { Incr } \\
2014-15\end{array}$ & $\begin{array}{c}\text { Incr } \\
2015-16\end{array}$ & $\begin{array}{c}\text { Incr } \\
2014-15\end{array}$ & $\begin{array}{c}\text { Incr } \\
2015-16\end{array}$ \\
\hline $\mathrm{T} 1$ & 28 & 26 & $-3,50 a$ & $33,15 a$ & $0,31 \mathrm{a}$ & $6,19 a$ \\
\hline $\mathrm{T} 2$ & 32 & 27 & 2,15 a & $56,37 \mathrm{~b}$ & $0,52 \mathrm{a}$ & $10,33 b$ \\
\hline
\end{tabular}

* Test LSD Fischer, $\alpha=0,05$. Letras distintas indican diferencias significativas

Dado el daño por animales se reforzó el cerco perimetral y durante la segunda evaluación fue posible verificar que existen diferencias en el desarrollo de las plantas, tanto en altura como en diámetro, con incrementos superiores para ambas variables.

Existe una diferencia significativa en el incremento en altura, con mayor desarrollo en las plantas protegidas con Tetrapak (T2) (Cuadro $\left.\mathrm{N}^{\circ} 10\right)$.

Esto mismo ocurre para la variable DAC, lo que significa que en general las plantas logran desarrollarse mejor cuando cuentan con protector de este tipo, por sobre el proyector de malla raschel que tiene una vida útil menor (Figura $\mathrm{N}^{\circ} 8$ ).

Respecto de la mortalidad, la tasa registrada fue distinta según tratamiento. temporadas.

Las plantas protegidas con Tetrapack (T2) muestran una supervivencia completa en dos

Las plantas protegidas con tela Raschel en tanto muestran supervivencia completa en la primera temporada (2014 - 2015), pero para la temporada 2015 - 2016 la supervivencia baja al $88,5 \%$.

Los resultados confirmarían así que la protección individual de plantas con Tetrapak resulta más eficiente que aquella con protectores confeccionados con malla Raschel y coligües, material este último que no ofreció adecuada protección en la segunda temporada ya que se degrada con el sol. 

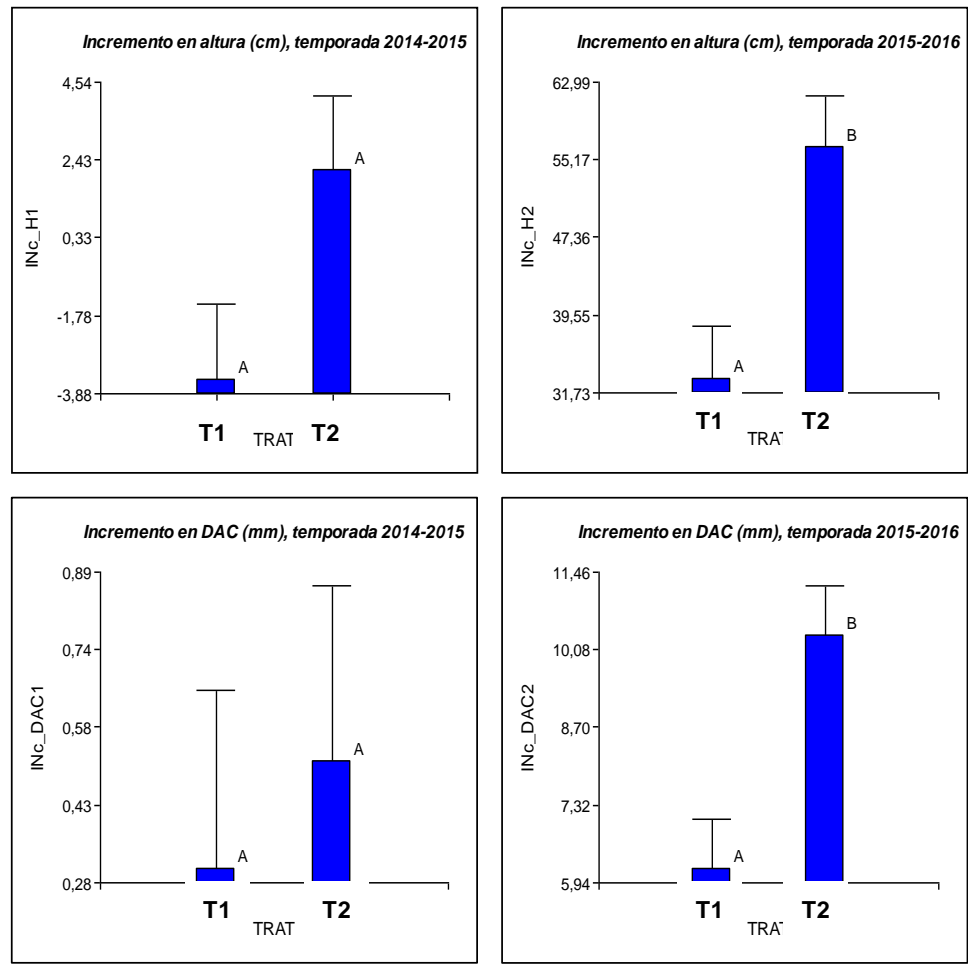

Figura $\mathbf{N}^{\circ} 8$

INCREMENTO EN ALTURA Y DAC SEGÚN TEMPORADA Y TIPO DE PROTECTOR

\section{Dispositivo Cocoon ${ }^{\circledR}$}

En el caso de los dispositivos Cocoon®, fueron establecidas plantas con una altura media de $17,1 \mathrm{~cm}$ y DAC medio de $6,2 \mathrm{~mm}$ (Cuadro $\mathrm{N}^{\circ} 11$ ). Las evaluaciones se realizaron en forma semestral registrando las variables altura y DAC, aun cuando la principal variable a evaluar es la supervivencia de las plantas establecidas con este nuevo sistema que incluye una reserva de agua.

Estos dispositivos contenedores de agua Cocoon® no sufrieron mayores daños, salvo uno de ellos cuya tapa colapsó, por lo que no fue considerado en la evaluación final.

Es posible apreciar que las plantas mantienen una supervivencia completa y una tasa de crecimiento importante. Los resultados indican que los dispositivos Cocoon $\circledast$ han cumplido con mantener el sustrato húmedo a pesar de no tener una fuente externa de agua, lográndose así la supervivencia de todas las plantas un año después del establecimiento del ensayo. 
Cuadro $\mathrm{N}^{\circ} 11$

VALORES MEDIOS DE VARIABLES EVALUADAS EN PLANTACIÓN CON COCOON®

\begin{tabular}{|c|c|c|c|c|c|c|c|c|c|}
\hline \multirow{2}{*}{ Tratamiento } & \multirow{2}{*}{$\begin{array}{c}\text { Plantas } \\
\text { Establecidas } \\
\left(\mathrm{N}^{\circ}\right)\end{array}$} & \multirow{2}{*}{$\begin{array}{c}\text { Plantas } \\
\text { Evaluadas } \\
\left(\mathrm{N}^{\circ}\right)\end{array}$} & \multicolumn{3}{|c|}{$\begin{array}{l}\text { Altura } \\
\mathrm{cm})\end{array}$} & \multicolumn{3}{|c|}{$\begin{array}{l}\text { DAC } \\
(\mathrm{mm})\end{array}$} & \multirow{2}{*}{$\begin{array}{c}\text { Mortalidad } \\
(\%)\end{array}$} \\
\hline & & & $\begin{array}{l}\text { Nov } \\
2016 \\
\end{array}$ & $\begin{array}{c}\text { Abr } \\
2017\end{array}$ & $\begin{array}{c}\text { Oct } \\
2017 \\
\end{array}$ & $\begin{array}{l}\text { Nov } \\
2016 \\
\end{array}$ & $\begin{array}{c}\text { Abr } \\
2017\end{array}$ & $\begin{array}{c}\text { Oct } \\
2017\end{array}$ & \\
\hline $\mathrm{T} 1$ & 52 & 51 & 17,1 & 52,9 & 51,2 & 6,2 & 9,0 & 9,7 & 0 \\
\hline
\end{tabular}
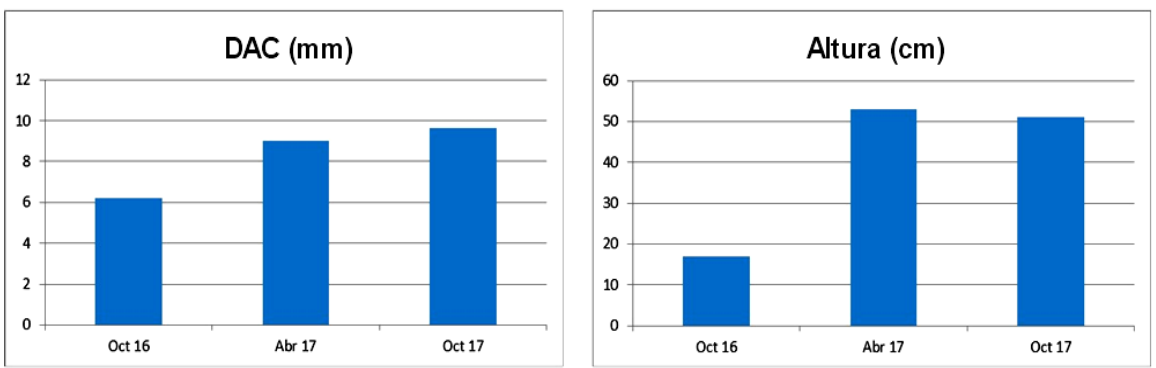

Figura $N^{\circ} 9$

VALORES MEDIOS VARIABLES EVALUADAS EN PLANTACIÓN CON COCOON®

\section{DISCUSIÓN Y CONCLUSIONES}

La fertilización inicial, al momento de la plantación, muestra un positivo efecto en el establecimiento de plantas de algarrobo, genera un mejoramiento en el crecimiento, tanto en altura como en DAC, con diferencias significativas con el tratamiento testigo en la primera temporada y manteniéndose estas diferencias en las dos temporadas siguiente aunque sin significancia estadística.

El ensayo establecido en 2013 recibió riegos periódicos hasta diciembre de 2014 y la supervivencia de plantas fue casi total en ambos tratamientos (con y sin fertilización inicial).

Mediciones posteriores deberán mostrar si este efecto se mantiene por más tiempo y si la supervivencia baja posteriormente en ausencia de riegos, dados los recurrentes períodos de sequía que caracterizan a la zona semiárida del país.

La fertilización inicial es una técnica a incorporar en el establecimiento de plantaciones de algarrobo y sería interesante ampliar esta investigación, variando la dosis de fertilizante aplicada y revisando por cuántas temporadas mantener el riego.

La protección individual de plantas aparece como una práctica indispensable para el establecimiento de plantaciones de algarrobo dado que la especie es altamente apetecida por animales. Caprinos, ovinos y otros animales domésticos pueden ser excluidos con un buen cerco perimetral, pero para lagomorfos y roedores silvestres se requiere de protección individual de las 
plantas, tal como quedó demostrado con los protectores probados, Tetrapack y malla Raschel, con mejores resultados para el primero. Sin embargo en cuanto las plantas excedieron la altura de los protectores fueron sistemáticamente ramoneados los ápices. Sería de interés ampliar esta investigación empleando otros tipos de protección individual y de mayor altura.

La utilización del dispositivo Cocoon® muestra muy buenos resultados, mantiene una supervivencia completa de plantas y un desarrollo adecuado en altura y DAC transcurrida una primera temporada de la plantación. Futuras evaluaciones permitirán apreciar si este efecto positivo se mantiene. Si es posible ampliar esta investigación sería interesante combinar el dispositivo Cocoon® con fertilización inicial de las plantas.

De los resultados de los tres ensayos se concluye que existen técnicas para el establecimiento de plantaciones de algarrobo que permiten mejorar sustantivamente el prendimiento de plantas y el desarrollo inicial de estas, y que su aplicación en plantaciones de mayor extensión sería un paso adelante para la recuperación y conservación del recurso que esta especie representa, tanto en términos ambientales como sociales y económicos.

Se debe continuar con la evaluación de estos ensayos y sería importante ampliar esta línea de investigación, con más variantes en materia de fertilización inicial y protección individual de plantas, e incorporar otras variables, como preparaciones de suelo más intensas (surco, subsolado), control de competencia de malezas y tiempo de permanencia de los riegos.

\section{REFERENCIAS}

Altamirano, G., 2012. Variedad de Frutos y Semillas en las Especies del Género Prosopis presentes en Chile. Corporación Nacional Forestal. Chile. Documento Técnico N²08. 12 p.

Balboa, O.; Parraguez, J. and Arce, P., 1988. Phenology Studies of Prosopis Species Growing in Chile. In: The Current State of Knowledge on Prosopis juliflora. II International Conference on Prosopis: Recife, Brazil, 25 - 29 August, 1986. Pp: 259-267

Bernuy, E., 2003. Incorporación de Harina y de Fibra Purificada del Fruto de Algarrobo (Prosopis chilensis (Mol) Stuntz) en el Desarrollo de Galletas. Tesis de Magíster en Ciencias Agropecuarias, Mención Producción Agroindustrial. Facultad de Ciencias Agronómicas, Universidad de Chile, Santiago, Chile. 82 p.

Caro, W.; Pinto, M. y Riveros, E., 1991. Utilización de Frutos de Algarrobo (Prosopis chilensis) para Reemplazar la Proteína en Dietas de Conejos de Carne. Avances en la Producción Animal. vol. 16 (1-2): 183-188.

González, M., 2013. Algarrobo. Rescatando una Especie de Alto Valor Histórico. Una Contribución a una Alimentación Sana y al Combate contra la Desertificación. Informe Técnico $N^{\circ} 195$, Instituto Forestal, Santiago, Chile. $128 p$.

INFOR, 1986. Estadísticas Forestales 1985. Serie Informática 34. División Estudios Económicos, Instituto Forestal, Corporación de Fomento de la Producción. Santiago, Chile. 98 p.

Martinez, B., 1998. Propuesta de Diseños Agroforestales. Ensayos de Adaptación de Especies Forestales. Proyecto FIA/CONAF. In: Actas Primer Congreso Latinoamericano IUFRO: EI Manejo Sustentable de los Recursos forestales: Un Desafío del Siglo XXI. 22 al 28 de noviembre 1998, Valdivia, Chile.

Valdivia, C. y Romero, C., 2013. En la Senda de la Extinción: El Caso del Algarrobo (Prosopis chilensis) (Fabaceae) y el Bosque Espinoso en la Región Metropolitana de Chile Central. Gayana Botánica 70 (1), versión On-line. 